PREPARED FOR THE U.S. DEPARTMENT OF ENERGY, UNDER CONTRACT DE-AC02-76CH03073

PPPL-4008

PPPL-4008

UC-70

Alfvén Continuum and Alfvén Eigenmodes in the National Compact Stellarator Experiment

by

O.P. Fesenyuk, Ya.I. Kolesnichenko, V.V. Lutsenko,

R.B. White, and Yu.V. Yakovenko

September 2004

NM|

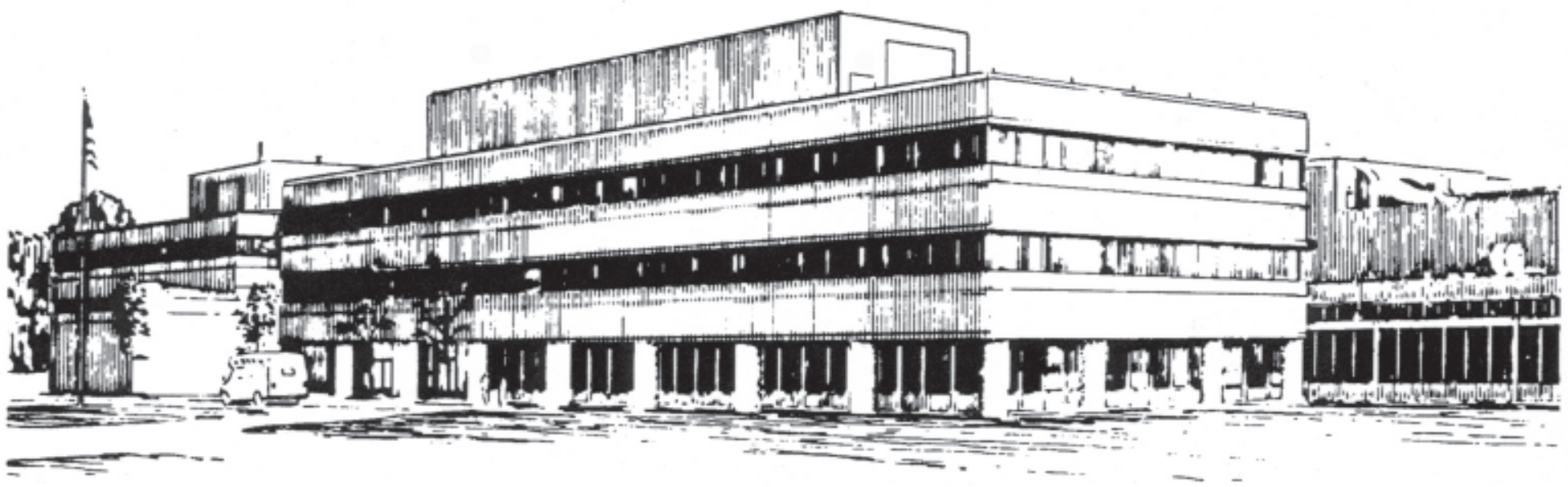

PRINCETON PLASMA PHYSICS LABORATORY PRINCETON UNIVERSITY, PRINCETON, NEW JERSEY 


\section{PPPL Reports Disclaimer}

This report was prepared as an account of work sponsored by an agency of the United States Government. Neither the United States Government nor any agency thereof, nor any of their employees, makes any warranty, express or implied, or assumes any legal liability or responsibility for the accuracy, completeness, or usefulness of any information, apparatus, product, or process disclosed, or represents that its use would not infringe privately owned rights. Reference herein to any specific commercial product, process, or service by trade name, trademark, manufacturer, or otherwise, does not necessarily constitute or imply its endorsement, recommendation, or favoring by the United States Government or any agency thereof. The views and opinions of authors expressed herein do not necessarily state or reflect those of the United States Government or any agency thereof.

\section{Availability}

This report is posted on the U.S. Department of Energy's Princeton Plasma Physics Laboratory Publications and Reports web site in Fiscal Year 2004. The home page for PPPL Reports and Publications is: http://www.pppl.gov/pub_report/

DOE and DOE Contractors can obtain copies of this report from:

U.S. Department of Energy

Office of Scientific and Technical Information

DOE Technical Information Services (DTIS)

P.O. Box 62

Oak Ridge, TN 37831

Telephone: (865) 576-8401

Fax: (865) 576-5728

Email: reports@adonis.osti.gov

This report is available to the general public from:

National Technical Information Service

U.S. Department of Commerce

5285 Port Royal Road

Springfield, VA 22161

Telephone: $1-800-553-6847$ or

(703) $605-6000$

Fax: (703) 321-8547

Internet: http://www.ntis.gov/ordering.htm 


\title{
Alfvén continuum and Alfvén eigenmodes in the National Compact Stellarator Experiment
}

\author{
O. P. Fesenyuk, Ya. I. Kolesnichenko, and V. V. Lutsenko \\ Institute for Nuclear Research, Prospekt Nauky 47, Kyiv 03680, Ukraine \\ R. B. White* \\ Princeton Plasma Physics Laboratory, \\ P.O. Box 451, Princeton, NJ, 08543, USA \\ Yu. V. Yakovenko \\ Institute for Nuclear Research, Prospekt Nauky 47, Kyiv 03680, Ukraine
}

\begin{abstract}
The Alfvén continuum (AC) in the National Compact Stellarator Experiment (NCSX) [G. H. Neilson et al., in Fusion Energy 2002, 19th Conference Proceedings, Lyon, 2002 (International Atomic Energy Agency, Vienna, 2003), Report IAEA-CN-94/IC-1] is investigated with the AC code COBRA [Ya. I. Kolesnichenko et al., Phys. Plasmas 8, 491 (2001)]. The resonant interaction of Alfvén eigenmodes and the fast ions produced by neutral beam injection is analyzed. Alfvén eigenmodes residing in one of the widest gap of the NCSX AC, the ellipticity-induced gap, are studied with the code BOA-E [V. V. Lutsenko et al., in Fusion Energy 2002, 19th Conference Proceedings, Lyon, 2002 (International Atomic Energy Agency, Vienna, 2003), Report IAEA-CN94-TH/P3-16].
\end{abstract}

PACS numbers: 52.35.Bj; 52.55.Hc; 52.65.Kj

Keywords: Stellarator; Alfvén continuum; Alfvén eigenmodes

*Electronic address: rwhite@pppl.gov 


\section{INTRODUCTION}

Shear Alfvén waves in stellarators attract considerable attention in recent years due to their ability to be destabilized by fast ions. Various Alfvén instabilities (AI) caused by beam ions have been observed in stellarators, ${ }^{1-3}$ and considerable losses of energetic ions during bursts of Alfvénic activity have been reported. ${ }^{3}$ Moreover, in the stellarator Wendelstein 7-AS ${ }^{4}$ such bursts sometimes lead to significant drops in the soft X-ray emission from the plasma ${ }^{3}$ which seems to evidence that the bulk plasma is also affected by the Alfvén instabilities. Even though in many cases such instabilities do not result in significant particle losses, studying them is still of practical interest as they can be a useful tool for the plasma diagnostics [so-called "MHD (magnetohydrodynamic) spectroscopy"]. ${ }^{5-7}$ In addition, there is an idea to use Alfvén eigenmodes excited by external antennae for removal of the helium ash from reactor plasmas. ${ }^{8}$

The diversity of the types of AIs possible in stellarators is rather wide. In particular, instabilities of the GAE modes ${ }^{9,10}$ (Global Alfvén Eigenmodes) have already been observed in stellarators. ${ }^{1}$ The so-called "gap" Alfvén eigenmodes reside in the frequency gaps that arise in the Alfvén continuum (AC) due to deviations of the magnetic configuration from the cylindrical symmetry. The modes that result from the poloidal asymmetry, namely, the TAE modes $^{11}$ (Toroidicity-induced Alfvén Eigenmodes), the EAE modes ${ }^{12}$ (Ellipticity-induced Alfvén Eigenmodes), and the NAE modes ${ }^{12}$ (Noncircular triangularity-induced Alfvén Eigenmodes), can exist both in tokamaks and in stellarators. Instabilities of TAE modes have already been identified in stellarator experiments, ${ }^{2,3}$ whereas instabilities of EAE and NAE modes have been observed in tokamaks, ${ }^{13,14}$ and there is no reason to deny that they can appear in stellarators, too. The toroidal asymmetry of the stellarators results in additional gaps, where the MAE modes ${ }^{15,16}$ (Mirror-induced Alfvén Eigenmodes) and numerous types of HAE modes ${ }^{15-18}$ (Helicity-induced Alfvén Eigenmodes) can reside. There are evidences that such modes have been observed in experiments. ${ }^{19-21}$

Non-ideal effects (the finite Larmor radius and plasma resistivity) are responsible for the existence of the kinetic counterparts of the mentioned gap modes, which have frequencies slightly above the corresponding AC gaps. In particular, KTAE ${ }^{22}$ (Kinetic TAE), $\mathrm{KMAE}^{23,24}$ (Kinetic MAE), and $\mathrm{KHAE}^{23}$ (Kinetic HAE) modes have been predicted theoretically; $\mathrm{KTAE}^{25}$ and $\mathrm{KEAE}^{26}$ modes have been observed in tokamak experiments. 
The main purpose of this work is to study the AC structure in the proposed stellarator NCSX $^{27}$ (National Compact Stellarator Experiment). NCSX is a relatively compact device exploiting the idea of the axial quasi-symmetry. ${ }^{28}$ In addition, this work is aimed at studying Alfvén Eigenmodes (AE) and the resonances that provide the interaction between beam ions and AEs.

Note that although the magnetic field in NCSX is quasi-axisymmetric, AC must have the "non-axisymmetric" gaps, like in other stellarators, because the NCSX plasma configuration is not axisymmetric in real space. The $\mathrm{AC}$ in one of the previous reference configurations of NCSX was calculated in a recent work with the code STELLGAP. ${ }^{29}$ In the present work, we use another code, the $\mathrm{AC}$ code $\mathrm{COBRA}^{15}$ (COntinuum BRanches of Alfvén waves), to study the AC in NCSX. The numerical algorithm implemented in COBRA makes it possible to resolve the structure of the continuum gaps with high accuracy. In particular, phenomena associated with crossings of continuum gaps and predicted in a recent work ${ }^{30}$ are revealed.

The structure of the work is as follows. In Sec. II, the basic equations are presented, the method of solution is outlined, and results of calculations of the AC in NCSX with the code COBRA are presented. In Sec. III, the resonances responsible for the possible destabilization of AEs in NCSX are analyzed, and EAE modes are calculated. Finally, the summary of the work is given in Sec. IV.

\section{ALFVÉN CONTINUUM}

\section{A. Basic equations and the method of solution}

The AC code COBRA solves the following equation describing the AC in a general toroidal configuration: ${ }^{15}$

$$
\hat{L}\left(g^{\psi \psi} \hat{L} \Phi\right)+\omega^{2} \frac{g^{\psi \psi} g B^{2}}{v_{A}^{2}} \Phi=0,
$$

where $\omega$ is the frequency; $\Phi$ is the wave function; $(\psi, \theta, \phi)$ are Boozer coordinates ${ }^{31}$ with $\psi$ the toroidal magnetic flux, $\theta$ and $\phi$ the poloidal and toroidal angles, respectively; $\hat{L}=$ $\partial / \partial \phi+\iota \partial / \partial \theta$ is an operator of differentiation along the field lines; $g^{\psi \psi}$ is the corresponding component of the contravariant metric tensor; $g$ is the metric tensor determinant; $v_{A}=$ $B /\left(4 \pi M_{i} n_{i}\right)$ is the Alfvén velocity; $B$ is the magnetic field strength; $M_{i}$ is the ion mass; $n_{i}=n_{i}(\psi)$ is the ion density. Equation (1) includes only derivatives by angles with $\psi$ 
playing the part of a parameter (actually, the equation includes only derivatives along a field line).

In the cylindrical geometry, when different harmonics of the wave are decoupled, AC consists of the corresponding branches $\omega=\omega_{m n}(\psi) \approx\left|k_{\|}\right| v_{A}$, where $k_{\|} \approx(m \iota-n) / R_{0}$ is the longitudinal wave number, $R_{0}=L / 2 \pi$ is the major radius, $L$ is the length of the magnetic axis. To see this, it is sufficient to recall that $g \approx R_{0}^{2} / B^{2}$ in Boozer coordinates (in a low-pressure, low-aspect-ratio plasma) and take $\Phi \propto \exp (\operatorname{im} \theta-i n \phi)$.

The coupling between different harmonics, which appears due to the angular dependence of the coefficients of Eq. (1), produces gaps in AC. When the coupling is not too strong, the $(\mu, \nu)$ Fourier harmonic of the magnetic configuration, i.e., the harmonic $\propto \exp (i \mu \theta-i \nu N \phi)$, produces a gap near the line

$$
\omega_{\mu \nu}^{*}(\psi) \approx|\mu \iota-\nu N| \frac{v_{A}}{2 R_{0}}
$$

on the plane $(\psi, \omega)$ [this line is the locus of the crossings of the pairs of cylindrical continuum branches $\omega=\omega_{m n}(\psi)$ and $\omega=\omega_{m+\mu, n+\nu N}(\psi)$ for arbitrary $m$ and $\left.n\right] .{ }^{15}$ We will refer to this gap as the $(\mu, \nu)$ gap and to $\mu$ and $\nu$ as the poloidal and toroidal coupling numbers, respectively. When the coupling is strong, which is typically the case in stellarators because of strong shaping, the interaction between the gaps displaces them, and their frequencies may sometimes differ from that given by Eq. (2) by a factor of two or even more.

In the code COBRA, the coefficients of Eq. (1) and the wave function are expanded in Fourier series. Noting that an arbitrary wave harmonic with the poloidal and toroidal mode numbers $(m, n)$ can interact only with harmonics with the mode numbers $(m+\mu, n+\nu N)$, where $\mu$ and $\nu$ are integers, $N$ is the number of the field periods, we can take $\Phi$ in the following form:

$$
\Phi=\sum_{p, s=-\infty}^{\infty} \Phi_{p s} \exp [i(m+p) \theta-i(n+s N) \phi] .
$$

Then Eq. (1) is reduced to an eigenvalue problem of the form

$$
\sum_{p, s=-\infty}^{\infty} \mathcal{G}_{p_{*}, s_{*} ; p, s}\left(\psi, \tilde{k}_{\|}\right) \Phi_{p s}=\omega^{2} \sum_{p, s=-\infty}^{\infty} \mathcal{C}_{p_{*}, s_{*} ; p, s}(\psi) \Phi_{p s}
$$

where $\mathcal{G}$ and $\mathcal{C}$ are infinite Hermitian matrices (see Ref. 15 for details), $\tilde{k}_{\|}=m \iota-n, \iota$ is the rotational transform. Note that $m$ and $n$ enter Eq. (4) only through the parameter $\tilde{k}_{\|}$, which is convenient for scanning the continuum. The code COBRA truncates Eq. (4) to a finite size and solves it with a standard solver. 
A key element of COBRA is the selection of the obtained eigensolutions. ${ }^{15}$ The matter is that the truncation of the matrices in Eq. (4) to a finite size is a source of error in the solutions, and the eigenvectors having large components with mode numbers near the truncation edge (i.e., with high mode numbers) are affected by the truncation very strongly. As a result, some part of the calculated spectrum never converges, no matter how large matrices are used in the calculations, unless the solutions "spoiled" by the truncation are excluded from the spectrum. This problem is unlikely to appear in calculations of tokamak spectra because the harmonics with different $n$ are decoupled, and the harmonics with very large poloidal wave numbers lie outside the spectral range of interest. As a result, taking a sufficiently large range in $m$, one can always secure a sufficient accuracy of all solutions within this range. In a stellarator $\mathrm{AC}$, continuum branches with high $m$ and $n$ can be found in any part of the spectrum (because $\omega_{m n}=|m \iota-n| v_{A} / R_{0}$ can be low for arbitrarily high $m$ and $n$ ). This means that for any finite matrix size the spectrum is "polluted" by "spoiled" solutions unless some selection of solutions is introduced. In COBRA, the selection strategy is the following. The code fixes a certain vector component that lies approximately in the center of the region of $(p, s)$ that are kept in Eq. (4) and selects several eigenvectors with the largest absolute value of this component. It is assumed that then the eigenvector components located near the edge of the region are small enough to secure a small truncation error. For instance, in the COBRA calculations presented below, only one or two "best" (in this sense) eigensolutions were selected for each analyzed matrix of the dimension from 259 to 451 . The price of this strategy in terms of the computation time is rather high, but our experience has shown that sufficient accuracy cannot be achieved unless we discard most obtained solutions. The convergence of the spectra was checked by comparison of the spectra obtained for different truncation ranges.

\section{B. Results of calculations}

For the analysis we took a 3-periodic configuration of NCSX with the major radius $R_{0}=1.49 \mathrm{~m}$ and the central magnetic field $B_{0}=1.56 \mathrm{~T}^{32}$ The configuration was calculated by a stellarator equilibrium code and was given in a file format adopted by the NCSX group. Accordingly, the code COBRA (the part responsible for processing the input data and calculating the metric tensor) was extended to accept files in this format. 
The most important harmonics of $g^{\psi \psi}$ for this configuration are presented versus $r / a$ [where $r=\left(2 \psi / B_{0}\right)^{1 / 2}$ is the radial coordinate, $a$ is the minor radius, i.e., $r$ at the plasma boundary] in Fig. 1. Here and below we use the following normalization for the harmonics of the metric tensor: ${ }^{15}$

$$
\begin{gathered}
g^{\psi \psi}=2 \bar{B} \psi \bar{\delta} h_{g}^{\psi \psi}, \quad g^{\psi \theta}=\bar{\delta} \bar{B} h_{g}^{\psi \theta}, \quad g^{\theta \theta}=\frac{\bar{\delta} \bar{B}}{2 \psi} h_{g}^{\theta \theta}, \\
h_{g}^{i j}=\delta^{i j}+\frac{1}{2} \sum_{\mu, \nu} \epsilon_{g(\mu, \nu)}^{i j} \exp (i \mu \theta-i \nu N \phi),
\end{gathered}
$$

where $i, j=\psi, \theta, \delta^{i j}$ is the Kronecker symbol, bars over quantities mean averaging in $\phi$ at the magnetic axis, $\delta=\left(\kappa+\kappa^{-1}\right) / 2, \kappa=\kappa(\phi)$ is the elongation of the plasma cross section. The quantities $\epsilon_{g(\mu, \nu)}^{i j}$ (normalized Fourier harmonics of metric tensor components) are functions of $\psi$ satisfying the relationship $\epsilon_{g(\mu, \nu)}^{i j}=\epsilon_{g(-\mu,-\nu)}^{i j *}$, where "*" means complex conjugate. Note that they are introduced so that $\epsilon_{g(0,0)}^{\psi \psi} \rightarrow 0$ and $\epsilon_{g(0,0)}^{\theta \theta} \rightarrow 0$ at $r \rightarrow 0$, and $\epsilon_{g(0,0)}^{\psi \theta} \equiv 0$.

As was mentioned above, NCSX is a quasi-axisymmetric ${ }^{28}$ (QAS) device: The magnetic field strength in Boozer coordinates is almost independent on $\phi$. As a result, the particle motion in such a device is almost like in a tokamak; in particular, there is an approximate constant of motion associated with the quasi-symmetry (the canonical angular momentum). However, the configuration is not symmetric in real space. As we observe in Fig. 1, the metric coefficient $g^{\psi \psi}$ has many significant harmonics that depend on $\phi$ (i.e., with $\nu \neq 0$ ). Therefore, one can expect that $\mathrm{AC}$ in quasi-axisymmetric stellarators is like in other stellarators rather than in tokamaks: It has numerous gaps associated with the toroidal asymmetry of the configuration (MAE and HAE gaps). Results of our calculations confirm this.

In our analysis we assume that the plasma consists of deuterium with the central ion density $n_{i 0}=10^{20} \mathrm{~m}^{-3}$ and consider several different ion density profiles. Note that the ion density enters Eq. (1) through $v_{A}$. As $n_{i}$ does not depend on $\theta$ and $\phi$, any variation in $n_{i}$ affects only the numerical magnitudes of the continuum frequencies $\left(\omega \propto n_{i}^{-1 / 2}\right)$ and does not influence the structure of the gaps.

The calculated $\mathrm{AC}$ for the ion density profile of the form $n_{i}(r)=n_{i 0}\left(1-0.99 r^{2} / a^{2}\right)^{1 / 2}$ is presented in Figs. 2 and 3. Black dots on this graph show AC obtained by solving Eq. (4) for several values of $r$, with $\tilde{k}_{\|}$scanned in a certain range. The matrix dimension in this calculation is 451, with 41 poloidal wave numbers and 11 toroidal wave numbers taken into 
account. The most important gaps are labeled by the respective coupling numbers, $\mu$ and $\nu$. One can see that the widest gaps are the $(\mu, \nu)=(2,1)$ gap and the $(2,0)$ gap, which are produced by the plasma elongation. In the central part of the plasma, the $(0,1),(4,1),(3,0)$ and $(4,2)$ gaps are also considerable. All these gaps result mainly from the corresponding harmonics of $g^{\psi \psi}$, except for the $(4,1)$ and $(4,2)$ gaps. From Fig. 8 one can see that the $(4,1)$ harmonic of the metric tensor is too small to account for the observed $(4,1)$ gap. The gap results mainly from the indirect coupling through the $(2,1)$ and $(2,0)$ harmonics of the plasma shape $[(4,1)=(2,1)+(2,0)$, see Ref. 15 for details]. The same applies to the $(4,2)$ gap, which is produced mainly by the $(2,1)$ harmonic of the shape $[(4,2)=2 \times(2,1)]$. The TAE gap $[(\mu, \nu)=(1,0)]$ is considerable only at the periphery, for $r<0.5 a$, which is one more evidence that the Alfvén spectra in QAS devices considerably differ from those in tokamaks.

Large grey dots in Fig. 2 are the results of a similar scan of AC for the matrix dimension of 259 (37 poloidal and 7 toroidal wave numbers), which are shown here in order to estimate the truncation error. Those parts of the continuum where the calculations have not converged (i.e., the achieved accuracy is not sufficient to resolve the gap structure, which can be seen from significant discrepancies between the results obtained with different matrix dimensions) are enclosed in frames; additional calculations with the matrices of a larger size are required to resolve these parts of the continuum. In particular, for some reasons the convergence is very poor between the $(0,1)$ and $(2,1)$ gaps. The accuracy is insufficient near the plasma boundary, too (except for low frequencies). The reason for this is that many harmonics of $g^{\psi \psi}$ and $B$ grow at the plasma periphery, which manifests itself in the increase of the widths of many gaps. As a result, more harmonics become efficiently coupled, and the size of the matrices taken here becomes too small. One can also notice that the convergence deteriorates near the radii where $\iota$ has the form $N / l$, where $l$ is an integer (for instance, near $r / a \approx 0.3$, where $\iota=3 / 7, r / a \approx 0.55$, where $\iota=3 / 6$, and $r / a \approx 0.8$, where $\iota=3 / 5)$. In addition, at such points the code usually makes mistakes in determining the gap boundaries. As will be shown below, this is accounted for by the influence of crossings of gaps.

The AC for a hollow density profile is shown in Fig. 4. The general structure of the continuum gaps is, as expected, the same as in Fig. 2. However, the shape of the gaps has changed, which may be of importance, e.g., for continuum damping.

The presence of crossings of considerable continuum gaps is an important feature of the 
NCSX AC, which is a consequence of relatively low $N$. We can conclude from Eq. (2) that two gaps with the coupling numbers $\left(\mu_{1}, \nu_{1}\right)$ and $\left(\mu_{2}, \nu_{2}\right)$, respectively, cross when the following relationship is satisfied:

$$
\mu_{1} \iota-\nu_{1} N= \pm\left(\mu_{2} \iota-\nu_{2} N\right)
$$

In LHD $^{33}$ (Large Helical Device, $\left.N=10, \iota \sim 1\right)$ and Wendelstein 7-AS $(N=5, \iota \sim 0.5)$, Eq. (7) can be satisfied only when $\mu_{1}$ or $\mu_{2}$ exceeds 5 , which means that, at least, one of the crossing gaps is rather narrow. In contrast to this, crossings of gaps with low coupling numbers are observed in NCSX. An example is the crossing of the $(3,0)$ and $(4,1)$ gaps, which lies at $r / a \approx 0.3(\iota=3 / 7)$ at the frequency about $200 \mathrm{kHz}$.

The behavior of AC near gap crossings was recently considered ${ }^{30}$ in connection with certain properties of AC in Wendelstein 7-AS. It was shown that the gaps "annihilate" at the crossing point, i.e., the width of the resulting gap at the crossing point is the difference of the widths of the crossing gaps. In particular, when two gaps of the same widths cross, they both disappear at the crossing point. To illustrate this, we have calculated the continuum near the crossing of the $(3,0)$ and $(4,1)$ gaps more accurately (see Fig. 5). For this calculation, 77 poloidal wave numbers and 11 toroidal wave numbers were taken (the dimension of the matrices was 847). The picture is similar to that obtained in Ref. 30 for a model configuration. As here we take into account all the configuration harmonics, the calculations are more cumbersome, and we failed to obtain as clear picture as in Ref. 30. However, main features are quite visible. First, the gaps indeed annihilate at the crossing (the net width of the two gaps near the crossing point is much less than the width of either gap near the edges of the interval). Second, multiple "combination" gaps appear [in this case, $(\mu, \nu)=(10,1)=(4,1)+2 \times(3,0),(11,2)=(3,0)+2 \times(4,1),(17,2)=2 \times(4,1)+3 \times(3,0)$ etc.]. It is also of importance that the continuum wave functions near the crossing point are combinations of numerous Fourier harmonics of approximately the same amplitude, which is associated with their strong angular localization. ${ }^{30}$ This explains why it is so difficult to obtain good numerical resolution of the continuum near gap crossings.

Finally, it is of interest to compare our results with the results of previous work. ${ }^{29}$ Figure 3 shows the same part of AC as Fig. 6 (b) of Ref. 29. The density profiles used for these two graphs are slightly different (the profile is parabolic in Ref. 29), and the abscissas of the graphs show different quantities $\left(r / a\right.$ here and the normalized toroidal flux $s=(r / a)^{2}$ in 
Ref. 29). Another difference between the graphs is that we show AC in general, without trying to identify particular AC branches with certain dominant mode numbers [except for the branches with $(m, n)=(-5,-2)$ and $(-3,-2)$ in Fig. 3, which will be discussed in Sec. III], whereas Ref. 29 gives several specific branches of AC. However, these differences must not complicate the comparison as the continuum gaps must have the same relative widths at a certain radius. The size and the frequency location of the $(2,0)$ (EAE) gap is approximately the same in Fig. 3 of the present work and in Fig. 6 (b) of Ref. 29, at least, in the plasma core (at the periphery the gap in Ref. 29 is much more narrow; e.g., for $r / a \approx 0.75 \div 0.85$, which corresponds to $s \approx 0.6 \div 0.7$, the relative width of the $(2,0)$ gap is about $15 \%$ in Ref. 29 and more than $30 \%$ in our calculations). However, even the widest $(2,1)$ gap is not visible in the latter picture, although specific "jumps" of curves above $300 \mathrm{kHz}$ indicate that it is there. The color coding shows that the gap is closed by continuum branches that lie at the boundary of the considered range of mode numbers and, thus, could not be calculated accurately. The same is true for the curves that account for the mentioned difference between the widths of the EAE gaps in the two graphs. Thus, the observed differences seem to be a result of insufficient selection of solutions in Ref. 29 .

\section{ALFVÉN EIGENMODES AND THEIR DESTABILIZATION BY ENER- GETIC IONS}

Using the calculated AC (Figs. 2-4), one can suppose that various types of discrete AEs, with the frequencies from $100 \mathrm{kHz}$ (EAE modes) to $900 \mathrm{kHz}$ (HAE 42 modes, where the subscript " 42 " refers to the coupling numbers) can exist. On the other hand, it folows from Fig. 3 that TAE modes can hardly exist or, at least, they can hardly be destabilized by the energetic ions because, first, the TAE gap is located at the plasma periphery and, therefore, TAEs are strongly damped because of the continuum damping and, second, the energeticparticle drive of TAEs is expected to be weak because the number of the energetic ions (and their spatial gradient) is expected to be small at the periphery. The frequency range between EAE and HAE in NCSX is more narrow than that in Wendelstein-line-stellarators and LHD, which is explained by the fact that $N$ in NCSX is smaller.

The source of energetic ions in NCSX is balanced tangential Neutral Beam Injection (NBI) of $50-\mathrm{keV}$ hydrogen atoms. This implies that circulating particles will play the main role 
in the destabilization of AEs, although the collisional pitch-angle scattering will produce a considerable amount of trapped particles with the energy $\mathcal{E} \ll 50 \mathrm{keV}$ (partly slowed-down particles), especially, when $z_{\text {eff }} \gg 1$ ( $z_{\text {eff }}$ is the effective charge number). Note that the axial symmetry of the magnetic field strength (in flux coordinates) leads to the absence of "non-axisymmetric" resonances of the wave-particle interaction, ${ }^{8}$ i.e., NCSX is a tokamaklike device from the point of view of the destabilization of Alfvén modes by energetic ions. However, a potential variety of AEs in the gaps of AC provides more characteristic resonant velocities of the energetic ions than in tokamaks. They $\operatorname{are}^{8}$

$$
\left|v_{\|}^{r}\right|=v_{A *}\left|1 \pm \frac{2 \iota_{*}}{\mu \iota_{*}-\nu N}\right|^{-1}
$$

where $r_{*}$ is the radius around which the mode is localized, $\mu$ and $\nu$ are the coupling numbers of the gap. Equation (8) is obtained in the assumption that the gap width in AC is negligible and the mode is well localized radially. The finite mode width and the finite gap width lead to other resonance velocities. In particular, the finite mode width can strongly enhance the instability providing the interaction of the mode and most energetic particles in the case when such interaction is not possible in the local approximation (for instance, particles with $v_{A} / 3 \ll v_{\|}<v_{A}$ can destabilize TAE modes). ${ }^{34}$ This fact may be of importance for the destabilization of AEs in NCSX, where $v_{b} / v_{A}=0.9\left(v_{b}=\sqrt{2 \mathcal{E}_{b} / M_{b}}, \mathcal{E}_{b}\right.$ the injection energy $)$ in the case of $M_{b}=M_{i}$, where $M_{b}$ and $M_{i}$ are the beam ion mass and the plasma ion mass, respectively. When making this estimate, we took $B=1.56 \mathrm{~T}, \mathcal{E}_{b}=50 \mathrm{keV}, n_{i}=10^{20} \mathrm{~m}^{-3}$.

Of course, the presence of gaps in AC does not guarantee the existence of weakly damped Alfvén waves that can be destabilized through the mentioned resonances. Therefore, below we seek for discrete AEs. We proceed from the following equation for Alfvén eigenmodes in low-pressure plasmas: ${ }^{15,35}$

$$
\omega^{2} \nabla \cdot\left(\frac{1}{v_{A}^{2}} \nabla_{\perp} \Phi\right)+B \nabla_{\|}\left\{\frac{1}{B^{2}} \nabla \cdot\left[B^{2} \nabla_{\perp}\left(\frac{1}{B} \nabla_{\|} \Phi\right)\right]\right\}=0,
$$

where $\Phi$ is the scalar potential of the wave, $\nabla_{\|}=\mathbf{b} \cdot \nabla, \mathbf{b}=\mathbf{B} / B, \nabla_{\perp}=\nabla-\mathbf{b} \nabla_{\|}$.

Considering an eigenmode residing in the $(\mu, \nu)$ gap, we assume that only two Fourier harmonics with the mode numbers $(m, n)=\left(m_{1}, n_{1}\right)$ and $(m, n)=\left(m_{2}, n_{2}\right)=\left(m_{1}+\mu, n_{1}+\right.$ $\nu N)$ are significant in the Fourier expansion of $\Phi$ :

$$
\Phi=\Phi_{1} \exp \left(i m_{1} \vartheta-i n_{1} \varphi\right)+\Phi_{2} \exp \left(i m_{2} \vartheta-i n_{2} \varphi\right)
$$


Substituting Eq. (10) to Eq. (9), keeping only the $\left(m_{1}, n_{1}\right)$ and $\left(m_{2}, n_{2}\right)$ harmonics of the resulting equation, and using Eqs. (5) and (6), we obtain two coupled equations for $\Phi_{1}$ and $\Phi_{2}$, which in the case of low aspect ratio look as follows:

$$
\begin{aligned}
& \frac{1}{r} \frac{d}{d r}\left\{r\left[\frac{\omega^{2}}{\bar{v}_{A}^{2}}\left(1+\frac{\epsilon_{c(0,0)}^{\psi \psi}}{2}\right)-k_{1}^{2}\left(1+\frac{\epsilon_{g(0,0)}^{\psi \psi}}{2}\right)\right] \frac{d \Phi_{1}}{d r}\right\} \\
& -\frac{m_{1}^{2}}{r^{2}}\left[\frac{\omega^{2}}{\bar{v}_{A}^{2}}\left(1+\frac{\epsilon_{c(0,0)}^{\theta \theta}}{2}\right)-k_{1}^{2}\left(1+\frac{\epsilon_{g(0,0)}^{\theta \theta}}{2}\right)\right] \Phi_{1}-\frac{k_{1}}{r}\left[r\left(1+\frac{\epsilon_{g(0,0)}^{\psi \psi}}{2}\right) k_{1}^{\prime}\right]^{\prime} \Phi_{1} \\
& +\frac{1}{2 r} \frac{d}{d r}\left[r\left(\frac{\omega^{2}}{\bar{v}_{A}^{2}} \epsilon_{c(\mu, \nu)}^{\psi \psi}-k_{1} k_{2} \epsilon_{g(\mu, \nu)}^{\psi \psi}\right) \frac{d \Phi_{2}}{d r}\right]-\frac{m_{1} m_{2}}{r^{2}}\left(\frac{\omega^{2}}{\bar{v}_{A}^{2}} \epsilon_{c(\mu, \nu)}^{\theta \theta}-k_{1} k_{2} \epsilon_{g(\mu, \nu)}^{\theta \theta}\right) \Phi_{2} \\
& -\omega^{2}\left[i \epsilon_{c(\mu, \nu)}^{\psi \theta} \frac{m_{1}+m_{2}}{2 r \bar{v}_{A}^{2}} \frac{d \Phi_{2}}{d r}+\frac{m_{2}}{2 r}\left(\frac{i \epsilon_{c(\mu, \nu)}^{\psi \theta}}{\bar{v}_{A}^{2}}\right)^{\prime} \Phi_{2}\right] \\
& +\frac{k_{1}}{2 r}\left[m_{1} i \epsilon_{g(\mu, \nu)}^{\psi \theta} \frac{d}{d r}\left(k_{2} \Phi_{2}\right)+m_{2} \frac{d}{d r}\left(i \epsilon_{g(\mu, \nu)}^{\psi \theta} k_{2} \Phi_{2}\right)\right] \\
& +\epsilon_{g(\mu, \nu)}^{\psi \psi} \frac{m_{2} n_{1}-m_{1} n_{2}}{2} \iota^{\prime} \frac{d \Phi_{2}}{d r}-\frac{k_{1}}{2 r} \frac{d}{d r}\left(r \epsilon_{g(\mu, \nu)}^{\psi \psi} k_{2}^{\prime}\right) \Phi_{2}=0, \\
& \frac{1}{r} \frac{d}{d r}\left\{r\left[\frac{\omega^{2}}{\bar{v}_{A}^{2}}\left(1+\frac{\epsilon_{c(0,0)}^{\psi \psi}}{2}\right)-k_{2}^{2}\left(1+\frac{\epsilon_{g(0,0)}^{\psi \psi}}{2}\right)\right] \frac{d \Phi_{2}}{d r}\right\} \\
& -\frac{m_{2}^{2}}{r^{2}}\left[\frac{\omega^{2}}{\bar{v}_{A}^{2}}\left(1+\frac{\epsilon_{c(0,0)}^{\theta \theta}}{2}\right)-k_{2}^{2}\left(1+\frac{\epsilon_{g(0,0)}^{\theta \theta}}{2}\right)\right] \Phi_{2}-\frac{k_{2}}{r}\left[r\left(1+\frac{\epsilon_{g(0,0)}^{\psi \psi}}{2}\right) k_{2}^{\prime}\right]^{\prime} \Phi_{2} \\
& +\frac{1}{2 r} \frac{d}{d r}\left[r\left(\frac{\omega^{2}}{\bar{v}_{A}^{2}} \epsilon_{c(\mu, \nu)}^{\psi \psi}-k_{1} k_{2} \epsilon_{g(\mu, \nu)}^{\psi \psi}\right) \frac{d \Phi_{1}}{d r}\right]-\frac{m_{1} m_{2}}{r^{2}}\left(\frac{\omega^{2}}{\bar{v}_{A}^{2}} \epsilon_{c(\mu, \nu)}^{\theta \theta}-k_{1} k_{2} \epsilon_{g(\mu, \nu)}^{\theta \theta}\right) \Phi_{1} \\
& +\omega^{2}\left[i \epsilon_{c(\mu, \nu)}^{\psi \theta} \frac{m_{1}+m_{2}}{2 r \bar{v}_{A}^{2}} \frac{d \Phi_{1}}{d r}+\frac{m_{1}}{2 r}\left(\frac{i \epsilon_{c(\mu, \nu)}^{\psi \theta}}{\bar{v}_{A}^{2}}\right)^{\prime} \Phi_{1}\right] \\
& -\frac{k_{2}}{2 r}\left[m_{2} i \epsilon_{g(\mu, \nu)}^{\psi \theta} \frac{d}{d r}\left(k_{1} \Phi_{1}\right)+m_{1} \frac{d}{d r}\left(i \epsilon_{g(\mu, \nu)}^{\psi \theta} k_{1} \Phi_{1}\right)\right] \\
& +\epsilon_{g(\mu, \nu)}^{\psi \psi} \frac{m_{1} n_{2}-m_{2} n_{1}}{2} \iota^{\prime} \frac{d \Phi_{1}}{d r}-\frac{k_{2}}{2 r} \frac{d}{d r}\left(r \epsilon_{g(\mu, \nu)}^{\psi \psi} k_{1}^{\prime}\right) \Phi_{1}=0,
\end{aligned}
$$

where $k_{i}=\left(m_{i} \iota-n_{i}\right) / R_{0}$ with $i=1,2 ; \bar{v}_{A}=\bar{B} /\left(4 \pi M_{i} n_{i}\right)^{1 / 2}$; the parameters $\epsilon_{c(\mu, \nu)}^{i j}$ are introduced by the relationships

$$
h_{c}^{i j} \equiv h_{g}^{i j} h^{-4}=\delta^{i j}+\frac{1}{2} \sum_{\mu, \nu} \epsilon_{c(\mu, \nu)}^{i j} \exp (i \mu \theta-i \nu N \phi)
$$

for $i, j=\psi, \theta ; h=B / \bar{B}$. 
We solve Eqs. (11) and (12) with the code BOA-E. ${ }^{20}$ Restricting ourselves to the lowfrequency part of the spectrum, we consider the case of EAEs $[(\mu, \nu)=(2,0)]$. The corresponding coupling parameters for the considered configuration are shown in Fig. 6. Calculations show that the modes do exist, at least, in the two-harmonic approximation used here. Figure 7 shows the calculated wave functions of eigenmodes with the mode numbers of the main harmonics being $(m, n)=(-5,-2)$ and $(m, n)=(-3,-2)$. The branches of AC with the dominant mode numbers $(m, n)=(-5,-2)$ and $(m, n)=(-3,-2)$ are shown in Fig. 3. We observe that the eigenfrequencies of the obtained eigenmodes do not cross these branches, which means that the continuum damping of the modes should not be strong. In addition, considering the $\mathrm{AC}$ branches calculated with only three wave harmonics taken into account (in the same Figure), we conclude that the effect of the wave harmonics omitted in the eigenmode calculations does not affect the qualitative behavior of the branches. These eigenmodes can be destabilized by the resonances $v_{\|}=v_{A}$ and $v_{\|}=v_{A} / 2$. The first resonance is possible due to the presence of the elliptical harmonic, $\epsilon_{B}^{(20)}$, and the diamagnetic harmonic, $\epsilon_{B}^{(00)}$, in the magnetic field strength, where $\epsilon_{B}^{(\mu \nu)}=B^{(\mu \nu)} / B_{0}$ when $\mu \neq 0$ or $\nu \neq 0, \epsilon_{B}^{(00)}=\left(B^{(00)}-B_{0}\right) / B_{0}, B^{(\mu \nu)}=B^{(\mu \nu)}(r)$ is the $(\mu, \nu)$ Fourier harmonic of $B$, $B_{0}=B^{(00)}(r=0)$. The second resonance is possible due to the toroidal harmonic, $\epsilon_{B}^{(10)}$. The toroidal harmonic dominates (see Fig. 8), but this does not mean that the resonance $v_{\|}=v_{A} / 2$ plays the main role. The matter is that the growth rate is roughly proportional to $\left|\epsilon_{B}^{(\mu \nu)}\right|^{2}\left|v_{\|}^{r}\right|^{7} f_{b}\left(v_{\|}^{r}\right)$, where $f_{b}=f_{b}(\mathbf{v})$ is the velocity distribution function of the beam ions [this can be seen from Eq. (37) of Ref. 8]. Because $\mathcal{E}_{c} \lesssim \mathcal{E}_{b}$, with $\mathcal{E}_{c} \sim T\left(M_{b} / M_{e}\right)^{1 / 3}$, the beam distribution function depends on $v$ considerably weaker than $v^{-3}$, which follows from $f_{b} \propto\left(v^{3}+v_{c}^{3}\right)^{-1}$, with $v_{c}=\sqrt{2 \mathcal{E}_{c} / M_{b}}$. Therefore, the first resonance will dominate during the injection of protons into a deuterium plasma (then $v_{b}>v_{A}$ ), whereas the second one will probably be main in the case of a hydrogen plasma (then $v_{b}<v_{A}$ and, thus, the resonance $v_{\|}=v_{A}$ is not so efficient).

\section{SUMMARY AND CONCLUSIONS}

We have calculated the Alfvén continuum in NCSX, using the code COBRA. It differs from that calculated in Ref. 29 by a better resolution of main gaps. Some parts of the continuum have not been resolved yet; extensive additional calculations with larger matrices 
are required to clarify these parts of the spectrum. It follows from the calculated AC that the destabilization of TAE modes by the beam ions with a peaked radial profile is hardly possible because the TAE gap is located at the plasma periphery. Another conclusion is that the gaps in AC associated with absence of the axial symmetry of the magnetic configuration (in real space) are located in a relatively low frequency range: In contrast to Wendelstein 7-AS and LHD, important HAE gaps reach the frequency range of the NAE, EAE and TAE gaps [for instance, the $(2,1)$ gap crosses the NAE gap, the $(4,1)$ gap crosses the NAE, EAE, and TAE gaps etc.]. This is explained by the low number of the field periods in NCSX $(N=3)$. As is shown, the gap crossings considerably affect the AC structure: The gap widths are strongly reduced at the radii corresponding to the crossings.

The existence of EAEs and the possibility of their destabilization by injected ions are investigated. In particular, with the use of the code BOA-E, it is found that there exist discrete EAEs in the lower part of the EAE gap, at least, in the approximation we use: The EAEs and the corresponding eigenfrequencies are calculated by solving a pair of coupled equations. These EAE modes can be destabilized by beam ions through the resonances $v_{\|}=v_{A}$ and $v_{\|}=v_{A} / 2$. The role of these resonances is different in the cases of the proton injection into hydrogen and deuterium plasmas.

\section{Acknowledgments}

The authors thank Dr. L. P. Ku for providing numerical data on an NCSX configuration.

The research described in this work was made possible in part by the Award No. UP22419-KV-02 of the U.S. Civilian Research \& Development Foundation and the Government of Ukraine and the U.S. Department of Energy under contract number DE-AC02-76-CH03073. 
1 A. Weller, D. A. Spong, R. Jaenicke, A. Lazaros, F. P. Penningsfeld, S. Sattler, W7-AS Team, and NBI group, Phys. Rev. Lett. 72, 1220 (1994).

2 K. Toi, M. Takechi, S. Ohdachi et al., in 5th International Atomic Energy Agency Technical Committee Meeting on Alpha Particles in Fusion Research, Abingdon, 1997 (International Atomic Energy Agency, Vienna, 1997), p. 197.

3 A. Weller, M. Anton, J. Geiger et al., Phys. Plasmas 8, 931 (2001).

${ }^{4}$ G. Grieger, W. Lotz, P. Merkel et al., Phys. Fluids B 4, 2081 (1992).

5 J. P. Goedbloed, H. A. Holties, S. Poedts, G. T. A. Huysmans, and W. Kerner, Plasma Phys. Control. Fusion 35, B277 (1993).

6 G. J. Kramer, M. Iwase, Y. Kusama et al., Nucl. Fusion 40, 1383 (2000).

7 A. Fasoli, D. Testa, S. Sharapov et al., Plasma Phys. Control. Fusion 44, B159 (2002).

8 Ya. I. Kolesnichenko, V. V. Lutsenko, H. Wobig, and Yu. V. Yakovenko, Phys. Plasmas 9, 517 (2002).

9 K. Appert, R. Gruber, F. Troyon, and J. Vaclavik, Plasma Phys. 24, 1147 (1982).

10 D. W. Ross, G. L. Chen, and S. M. Mahajan, Phys. Fluids 25, 652 (1982).

11 C. Z. Cheng and M. S. Chance, Phys. Fluids 29, 3695 (1986).

12 J. R. Betti and J. P. Freidberg, Phys. Fluids B 4, 1465 (1992).

13 A. D. Turnbull, E. J. Strait, W. W. Heidbrink et al., Phys. Fluids B 5, 2546 (1993).

14 G. J. Kramer, M. Saigusa, T. Ozeki et al., Phys. Rev. Lett. 80, 2594 (1998).

15 Ya. I. Kolesnichenko, V. V. Lutsenko, H. Wobig, Yu. V. Yakovenko, and O. P. Fesenyuk, Alfvén Eigenmodes in Helias Configurations (Part I), IPP Report III/261 (Max-Planck-Institut für Plasmaphysik, Garching bei München, May 2000); Phys. Plasmas 8, 491 (2001).

16 C. Nührenberg, in ISSP-19 "Piero Caldirola", Theory of Fusion Plasmas, edited by J. W. Connor, O. Sauter, and E. Sindoni (Editrice Compositori - Società Italiana di Fisica, Bologna, 2000), p. 313.

17 N. Nakajima, C. Z. Cheng, and M. Okamoto, Phys. Fluids B 4, 1115 (1992).

18 C. Nührenberg, Plasma Phys. Control. Fusion 41, 1055 (1999).

19 S. Yamamoto, K. Toi, S. Ohdachi et al., in 29th European Physical Society Conference on Controlled Fusion and Plasma Physics, Montreux, 2002, Europhysics Conference Abstracts, 
Vol. 26B (European Physical Society, Petit-Lancy, 2002), Report P1-079.

20 V. V. Lutsenko, Ya. I. Kolesnichenko, A. Weller, A. Werner, H. Wobig, Yu. V. Yakovenko, and O. P. Fesenyuk in Fusion Energy 2002, 19th Conference Proceedings, Lyon, 2002 (International Atomic Energy Agency, Vienna, 2003), Report IAEA-CN-94-TH/P3-16.

21 A. Weller, J. Geiger, A. Werner et al., Plasma Phys. Control. Fusion, 45, A285 (2003).

22 R. R. Mett and S. M. Mahajan, Phys. Fluids B 4, 2885 (1992).

23 O. P. Fesenyuk, Ya. I. Kolesnichenko, V. V. Lutsenko, H. Wobig, and Yu. V. Yakovenko, in 30th European Physical Society Conference on Controlled Fusion and Plasma Physics, St. Petersburg, 2003, Europhysics Conference Abstracts, Vol. 27A (European Physical Society, Petit-Lancy, 2003), Report P-3.4.

24 O. P. Fesenyuk, Ya. I. Kolesnichenko, V. V. Lutsenko, H. Wobig, and Yu. V. Yakovenko, Plasma Phys. Control. Fusion 46, 89 (2004).

25 A. Fasoli, J. B. Lister, S. Sharapov et al., Phys. Rev. Lett. 76, 1067 (1996).

26 R. F. Heeter, A. Fasoli, D. Borba, D. Start, S. Ali-Arshad, and N. Fisch, in 5th International Atomic Energy Agency Technical Committee Meeting on Alpha Particles in Fusion Research, Abingdon, 1997 (International Atomic Energy Agency, Vienna, 1997), p. 9.

27 G. H. Neilson, M. Zarnstorff, L. P. Ku et al., in Fusion Energy 2002, 19th Conference Proceedings, Lyon, 2002 (International Atomic Energy Agency, Vienna, 2003), Report IAEA-CN94/IC-1.

28 J. Nührenberg, W. Lotz, and S. Gori, in Theory of Fusion Plasmas, Varenna, 1994 (Editrice Compositori, Bologna, 1994), p. 3.

29 D. A. Spong, R. Sanchez, and A. Weller, Phys. Plasmas 10, 3217 (2003).

30 Yu. V. Yakovenko, Ya. I. Kolesnichenko, V. V. Lutsenko, A. Weller, A. Werner, S. Zegenhagen, J. Geiger, and A. Dinklage, "Confinement relevant Alfvén instabilities in Wendelstein 7-AS", 20th International Atomic Energy Agency Fusion Energy Conference, Vilamoura, November 2004, Paper TH/P4-48, to be presented.

31 A. H. Boozer, Phys. Fluids 24, 1999 (1981).

32 L. P. Ku, private communication (2002).

33 M. Fujiwara, K. Kawahata, N. Ohyabu et al., Nucl. Fusion 41, 1355 (2001).

34 Ya. I. Kolesnichenko, S. Yamamoto, K. Yamazaki, V. V. Lutsenko, N. Nakajima, Y. Narushima, K. Toi, and Yu. V. Yakovenko, Phys. Plasmas 11, 158 (2004). 
35 J. Candy, B. N. Breizman, J. W. Van Dam, and T. Ozeki, Phys. Lett. A 215, 299 (1996). 


\section{Figures}

FIG. 1: Normalized Fourier harmonics of $g^{\psi \psi}$. Each curve is labeled by the corresponding coupling numbers, $(\mu, \nu)$.

FIG. 2: The Alfvén continuum for the ion density profile $n_{i}(r)=n_{i 0}\left(1-0.99 r^{2} / a^{2}\right)^{1 / 2}, n_{i 0}=$ $10^{20} \mathrm{~m}^{-3}$. Notations: black dots, $\mathrm{AC}$ at certain radial points; large grey dots, the same continuum but calculated with a smaller matrix size; solid lines, calculated boundaries of some gaps. The gaps are labeled by the corresponding coupling numbers, $(\mu, \nu)$. The frames encircle parts of the continuum where the calculations have not converged.

FIG. 3: The low-frequency part of the Alfvén continuum shown in Fig. 2. Empty circles and triangles show the continuum branches with the mode numbers, $(m, n)=(-5,-2)$ and $(-3,-2)$, respectively; filled circles and triangles, the same branches but calculated with only the harmonics $(-5,-2),(-4,-2)$, and $(-3,-2)$ taken into account.

FIG. 4: The same as Fig. 2 but for a hollow density profile, $n_{i}(r)=n_{i 0}\left(1-0.99 r^{2} / a^{2}\right) /[1-$ $\left.r^{2} /(0.8 a)^{2}\right]^{2}$.

FIG. 5: A part of the continuum shown in Fig. 2 near the crossing of the $(3,0)$ and $(4,1)$ gaps.

FIG. 6: Normalized $(2,0)$ Fourier harmonics of the metric tensor and the magnetic field strength.

FIG. 7: The wave functions of EAE modes with the mode numbers of the dominant harmonics $(-5,-2)$ and $(-3,-2)$. Solid line corresponds to the eigenfrequency of $134 \mathrm{kHz}$; dotted line, $121 \mathrm{kHz}$.

FIG. 8: Normalized dominant Fourier harmonics of the magnetic field strength. Each curve is labeled by the corresponding coupling numbers, $(\mu, \nu)$. 


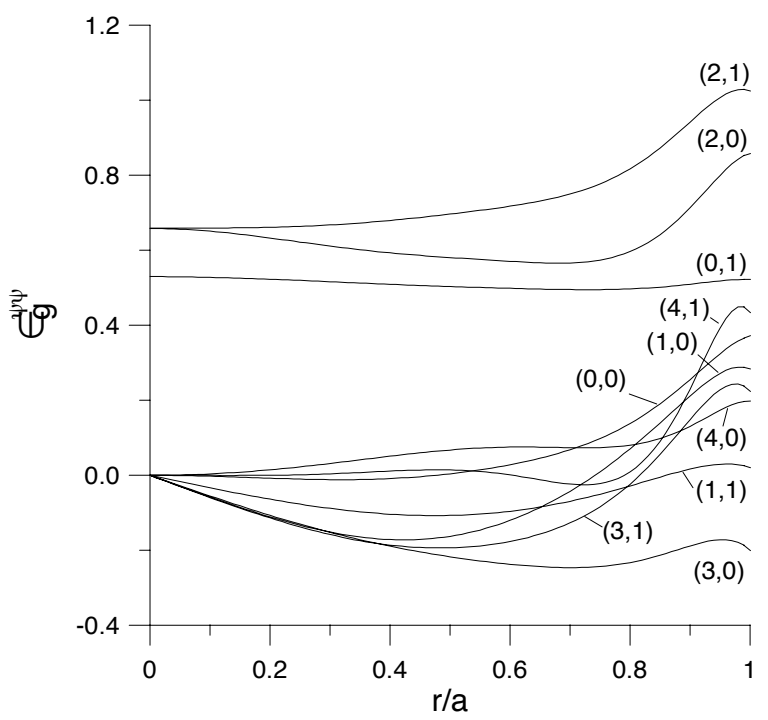

FIG. 1 "Alfvèn continuum and Alfvèn eigenmodes in the the National Compact Stellarator Experiment" by O. P. Fesenyuk et al. 


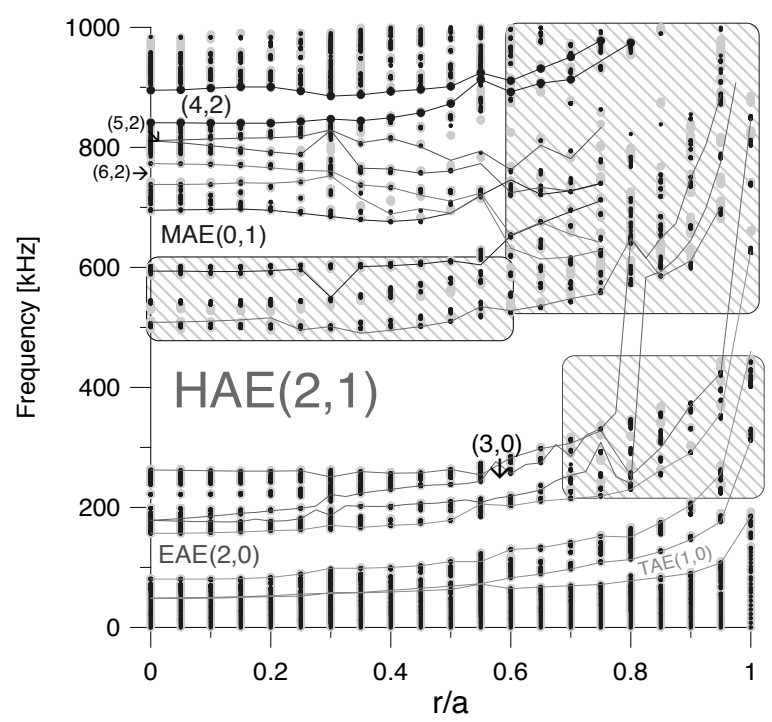

FIG. 2 "Alfvèn continuum and Alfvèn eigenmodes in the the National Compact Stellarator Experiment" by O. P. Fesenyuk et al. 


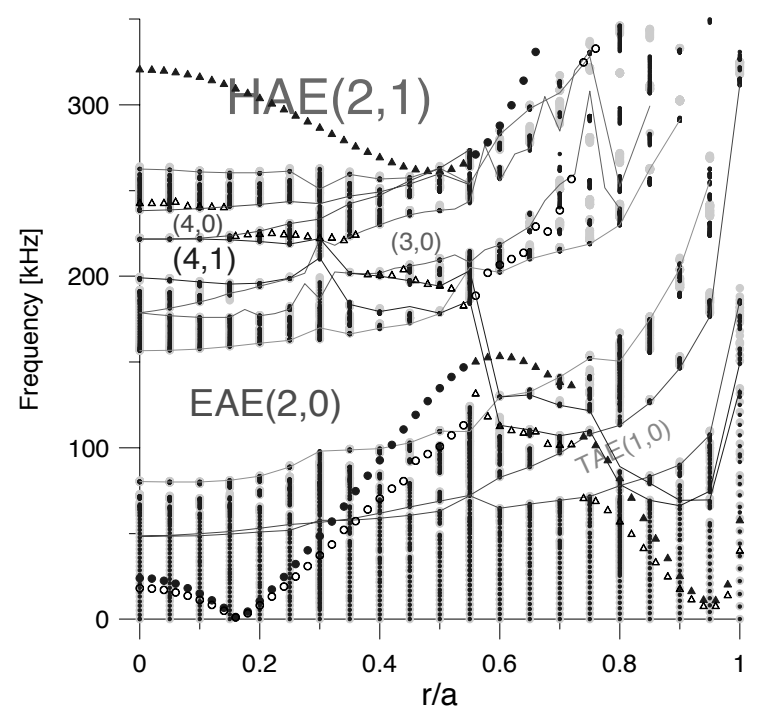

FIG. 3 "Alfvèn continuum and Alfvèn eigenmodes in the the National Compact Stellarator Experiment" by $\mathrm{O}$. P. Fesenyuk et al. 


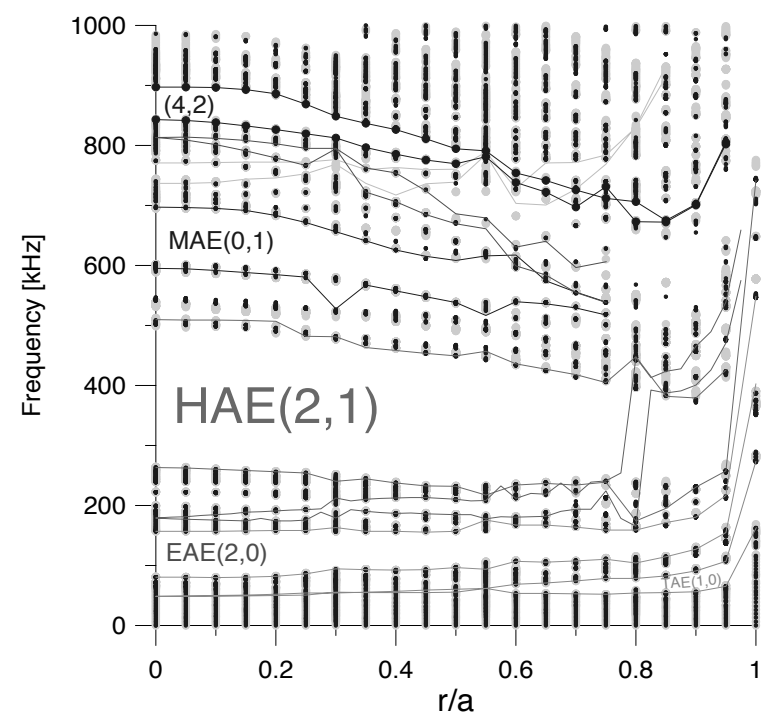

FIG. 4 "Alfvèn continuum and Alfvèn eigenmodes in the the National Compact Stellarator Experiment" by O. P. Fesenyuk et al. 


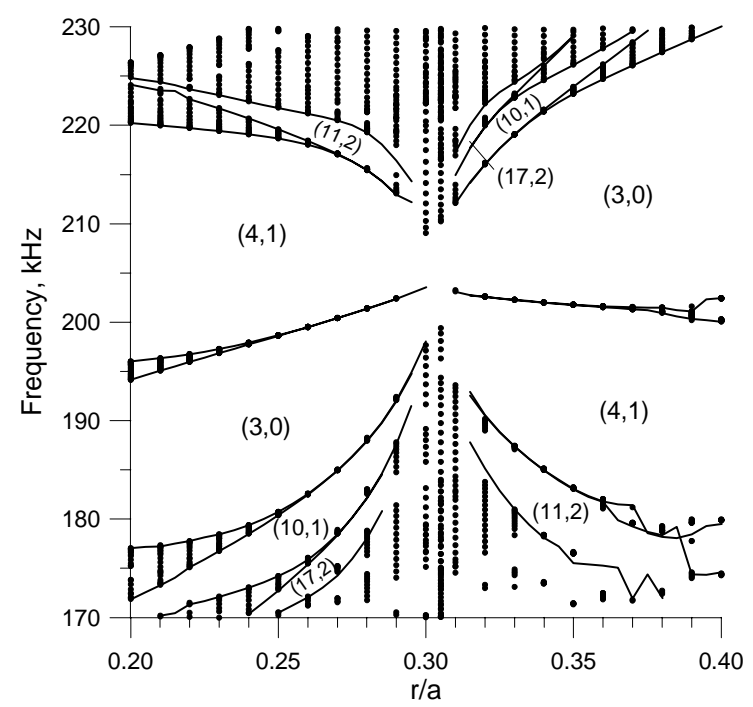

FIG. 5.

"Alfven continuum and Alfven eigenmodes in the National Compact Stellarator Experiment" by O.P. Fesenyuk et al. 


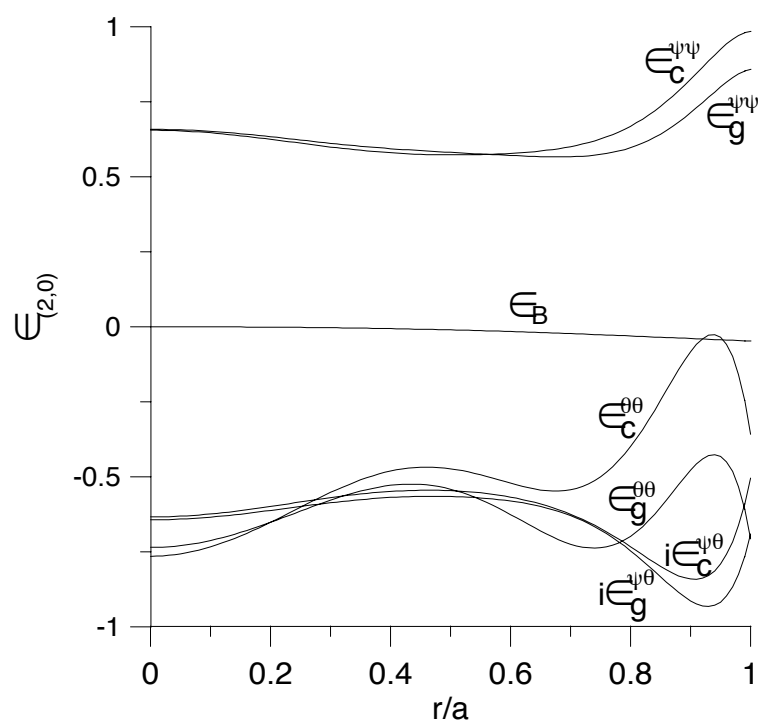

FIG. 6 "Alfvèn continuum and Alfvèn eigenmodes in the the National Compact Stellarator Experiment" by O. P. Fesenyuk et al. 
Fig.7.

Alfvén continuum and Alfvén eigenmodes in National Compact Stellarator eXperiment O.P. Fesenyuk, Ya.I. Kolesnichenko, V.V. Lutsenko, Yu.V. Yakovenko, R.B. White

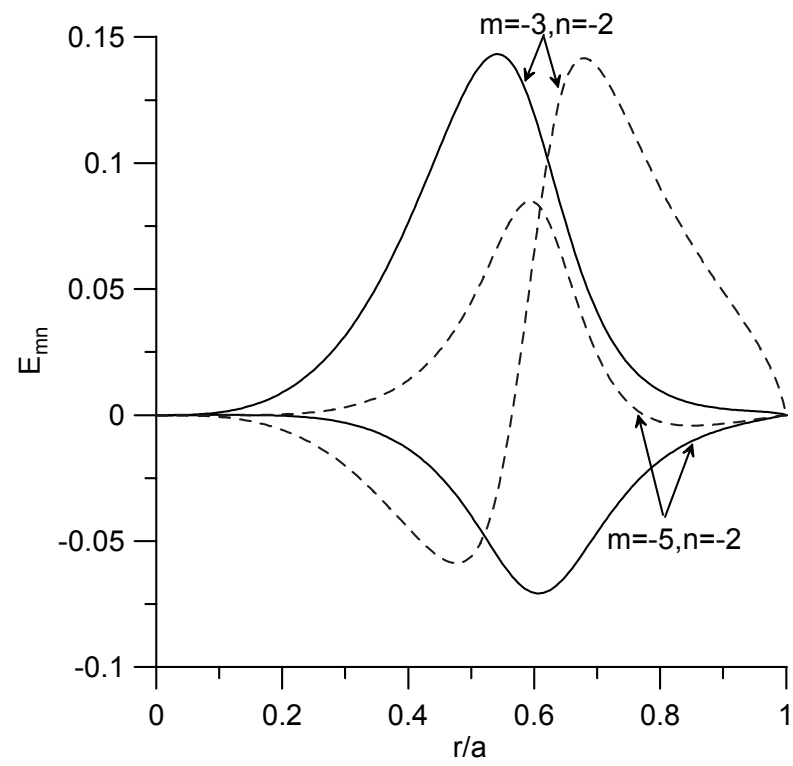




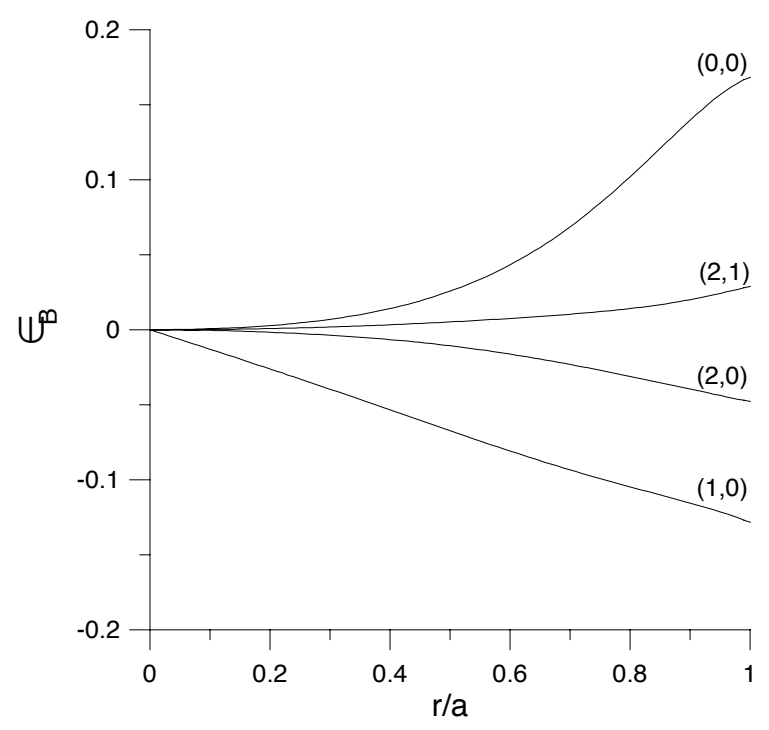

FIG. 8 "Alfvèn continuum and Alfvèn eigenmodes in the the National Compact Stellarator Experiment" by O. P. Fesenyuk et al. 



\section{External Distribution}

Plasma Research Laboratory, Australian National University, Australia

Professor I.R. Jones, Flinders University, Australia

Professor João Canalle, Instituto de Fisica DEQ/IF - UERJ, Brazil

Mr. Gerson O. Ludwig, Instituto Nacional de Pesquisas, Brazil

Dr. P.H. Sakanaka, Instituto Fisica, Brazil

The Librarian, Culham Laboratory, England

Mrs. S.A. Hutchinson, JET Library, England

Professor M.N. Bussac, Ecole Polytechnique, France

Librarian, Max-Planck-Institut für Plasmaphysik, Germany

Jolan Moldvai, Reports Library, Hungarian Academy of Sciences, Central Research Institute for Physics, Hungary

Dr. P. Kaw, Institute for Plasma Research, India

Ms. P.J. Pathak, Librarian, Institute for Plasma Research, India

Ms. Clelia De Palo, Associazione EURATOM-ENEA, Italy

Dr. G. Grosso, Instituto di Fisica del Plasma, Italy

Librarian, Naka Fusion Research Establishment, JAERI, Japan

Library, Laboratory for Complex Energy Processes, Institute for Advanced Study, Kyoto University, Japan

Research Information Center, National Institute for Fusion Science, Japan

Dr. O. Mitarai, Kyushu Tokai University, Japan

Dr. Jiangang Li, Institute of Plasma Physics, Chinese Academy of Sciences, People's Republic of China

Professor Yuping Huo, School of Physical Science and Technology, People's Republic of China

Library, Academia Sinica, Institute of Plasma Physics, People's Republic of China

Librarian, Institute of Physics, Chinese Academy of Sciences, People's Republic of China

Dr. S. Mirnov, TRINITI, Troitsk, Russian Federation, Russia

Dr. V.S. Strelkov, Kurchatov Institute, Russian Federation, Russia

Professor Peter Lukac, Katedra Fyziky Plazmy MFF UK, Mlynska dolina F-2, Komenskeho Univerzita, SK-842 15 Bratislava, Slovakia

Dr. G.S. Lee, Korea Basic Science Institute, South Korea

Institute for Plasma Research, University of Maryland, USA

Librarian, Fusion Energy Division, Oak Ridge National Laboratory, USA

Librarian, Institute of Fusion Studies, University of Texas, USA

Librarian, Magnetic Fusion Program, Lawrence Livermore National Laboratory, USA

Library, General Atomics, USA

Plasma Physics Group, Fusion Energy Research Program, University of California at San Diego, USA

Plasma Physics Library, Columbia University, USA

Alkesh Punjabi, Center for Fusion Research and Training, Hampton University, USA

Dr. W.M. Stacey, Fusion Research Center, Georgia Institute of Technology, USA

Dr. John Willis, U.S. Department of Energy, Office of Fusion Energy Sciences, USA

Mr. Paul H. Wright, Indianapolis, Indiana, USA 
The Princeton Plasma Physics Laboratory is operated by Princeton University under contract with the U.S. Department of Energy.

\author{
Information Services \\ Princeton Plasma Physics Laboratory \\ P.O. Box 451 \\ Princeton, NJ 08543
}

Phone: 609-243-2750

Fax: 609-243-2751

e-mail: pppl_info@pppl.gov

Internet Address: http://www.pppl.gov 